\title{
Influences relatives des agents hydrodynamiques sur la mobilité sédimentaire des Pertuis Charentais
}

Déborah Idier $^{(1)}$, Rodrigo Pedreros ${ }^{(1)}$, Carlos Oliveros ${ }^{(1)}$, Aldo Sottolichio $^{(2)}$ et Xavier Bertin ${ }^{(3)}$

(1) BRGM, Service Aménagement et Risques Naturels, 3 av. C. Guillemin, BP 6009, 45060 Orléans cedex 2, d.idier@brgm.fr

${ }^{(2)}$ EPOC - UMR 5805 - Université Bordeaux I, avenue des Facultés, 33405 Talence.

${ }^{(3)}$ Centre Littoral de Géophysique - Université de La Rochelle, av. M. Crépeau, 17042 La Rochelle cedex 1.

\begin{abstract}
Résumé
L'influence relative des vagues, courants de marée et courants induits par le vent sur la mobilité sédimentaire est étudiée en domaine de plateforme interne macrotidale (au large des Pertuis Charentais), en s'appuyant sur des mesures in-situ. Ces mesures, réalisées par $23 \mathrm{~m}$ de fond, montrent que les houles (plutôt que la mer de vent) permettent la mise en mouvement des sables, qui peuvent ensuite être transportés par les courants. Les sables de $0,2 \mathrm{~mm}$ seraient ainsi transportés pendant $92 \%$ de la durée de la mesure, tandis que les graviers seraient sporadiquement mis en mouvement, lors de conjonctions vive-eau - tempête.
\end{abstract}

\begin{abstract}
This paper investigates the relative influence of waves, wind-induced current and tidal current on the sediment mobility of a macro-tidal environnement belonging to the inner shelf seaward of the Pertuis Charentais (France). This study, mainly based on hydrodynamic in-situ measurements, shows that, for a water depth of 23 $\mathrm{m}$ water depth, the swell (rather than wind-waves) orbital velocity is large enough to initiate motion of medium sands which is then transported by currents. Estimations show that medium sand of $0.2 \mathrm{~mm}$ is transported during $92 \%$ of the measurements, whereas gravel moves sporadically, during spring tides synchronous with storm.
\end{abstract}

Mots-clé : Pertuis Charentais, courants, vagues, plateau continental, mobilité, sédiment.

\section{Introduction}

Les fonds marins, en particulier dans les zones de plateforme continentale interne, sous régime macro-tidal, sont soumis à l'action des courants de marée et de la houle [9]. L'analyse des processus de transport sédimentaire passe par l'estimation de l'influence de chacun de ces deux processus. L'impact relatif théorique de la houle et des courants de marée sur les sédiments de la plate-forme 
du Golfe de Gascogne a déjà fait l'objet d'une étude [2], basée sur des mesures hydrodynamiques d'une part, et des abaques de mise en mouvement et de transport de sédiments d'autre part. Cette étude a permis d'apporter des précisions sur la mobilité sédimentaire à l'échelle du Golfe. Toutefois, selon les auteurs, elle comporte quelques limites, avec, en particulier, une surestimation de la mobilité des sédiments, d'après des traçages radioactifs.

Le but de ce travail est, à partir de mesures hydrodynamiques, en affinant l'approche utilisée dans [2], d'étudier l'influence des composantes de courants de marée, de houle, de mer de vent et de courants résiduels sur la mise en mouvement et le transport des sédiments. Une méthodologie complète d'analyse des mesures hydrodynamiques est ainsi appliquée à un site de la plateforme interne du Golfe de Gascogne d'un estuaire : le Pertuis d'Antioche [6].

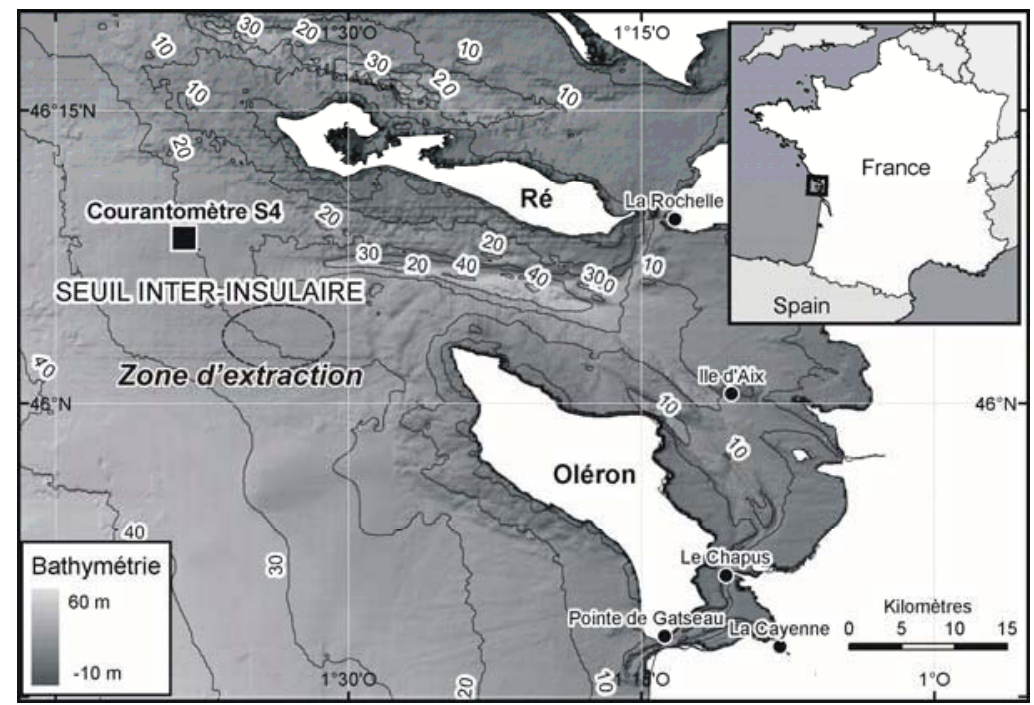

Figure 1 - Zone d'étude (seuil interinsulaire) et localisation du courantomètre S4. MNT obtenu à partir de données SHOM et IFREMER.

\section{Zone d'étude et données}

\subsection{Zone d'étude}

Le site d'étude, à savoir le seuil inter-insulaire, au large des Pertuis d'Antioche, a été choisi car c'est un secteur d'extraction de granulats (Fig. 1) qui est sujet à des phénomènes d'érosion [15] et qui a déjà fait l'objet de nombreuses études sédimentaires [3,15]. Située sur la côte Atlantique (Fig. 1), cette baie est délimitée par l'île de Ré et l'île d'Oléron. D'après le modèle de courants [12], les courants de marée intégrés sur la verticale sont de l'ordre de $0,45 \mathrm{~m} / \mathrm{s}$ et le marnage est de 5,1 m en vive-eau moyenne au niveau du seuil interinsulaire. Cette zone est recouverte de sable fin, et localement de sables graveleux. A partir d'observations sédimentaires, Weber et al. [15] émettent l'hypothèse suivante, que nous tenterons de vérifier : le seuil inter-insulaire serait un domaine en érosion, dominé par l'action des vagues. 


\subsection{Campagne de mesure in-situ, données externes et contexte hydro- météorologique}

Les mesures hydrodynamiques présentées dans cet article ont été réalisées dans le cadre d'une campagne «BRGM- UMR EPOC - Univ. La Rochelle » (Fig. 1, point S4). Un courantomètre électromagnétique S4 ADW a été mouillé par $23 \mathrm{~m}$ de fond, du 5 au 27 novembre 2002. Ce capteur fournit des mesures de pression et vitesse, $40 \mathrm{~cm}$ au-dessus du fond, à une fréquence de $2 \mathrm{~Hz}$.

Nos résultats sont systématiquement comparés à des données externes, provenant de modèles déjà validés ou de mesures indépendantes. Les sources de données externes, sont: Wavewatch III (houle, vent, au point le plus proche au large du point $\mathrm{S} 4\left(46^{\circ} \mathrm{N} ; 2,5^{\circ} \mathrm{W}\right)$, utilisé pour estimer et analyser les courants induits par le vent), COAMPS-Europe (vent, au point $\left(46,2^{\circ} \mathrm{N} ; 1,6^{\circ} \mathrm{W}\right)$, utilisé pour discriminer la houle de la mer de vent), SHOM (marée à La Rochelle et composantes harmoniques de marée près du courantomètre $S 4$ [8], utilisé pour valider nos traitements).

Pendant la campagne, les coefficients de marée étaient compris entre 36 et 112 cycle vive-eau - morte-eau. Trois épisodes de tempêtes ont eu lieu (Fig. 2a) : le 07, les 13-14 et les 20-21, avec des vitesses de vent atteignant $15-20 \mathrm{~m} / \mathrm{s}$. Pendant cette période, la hauteur significative des vagues, obtenue après traitement de la mesure S4, atteint plus de $6 \mathrm{~m}$, pour une période pic de $18 \mathrm{~s}$ (Fig. 2d,e).

\section{Méthode}

\subsection{Traitement des mesures hydrodynamiques}

La mesure du courantomètre S4 est traitée pour dissocier signal de marée, signal résiduel (i.e. signal brut moins le signal de houle moins le signal de marée), houle et mer de vent.

Afin d'estimer les composantes de marée et composantes résiduelles, le signal oscillatoire lié aux vagues est filtré en appliquant un filtre passe-bas à 1/120 Hz. L'estimation de la composante de marée est effectuée par analyse des composantes harmoniques (logiciel T_Tide [10]). Une fois les composantes de marée extraites (hauteur d'eau $h$, composantes de vitesse), le signal est recomposé en signal temporel. Le courant résiduel est estimé par différence entre le courant total (issu du filtrage passe-bas à $1 / 120 \mathrm{~Hz}$ ) et le courant de marée (issu du traitement par composantes harmoniques).

Afin d'estimer les caractéristiques des vagues, le signal brut est traité par analyse spectrale directionnelle sur des échantillons de $30 \mathrm{~min}$, à l'aide d'une boîte à outil développée à partir de la boîte à outil Diwasp [7], en intégrant un algorithme de partition du spectre [4]. La méthode d'analyse spectrale est celle de l'entropie maximale étendue [5]. Une fonction de transfert $(K p)$, basée sur la théorie linéaire de la houle est appliquée, pour tenir compte des effets non hydrostatiques : $K p=\cosh (k z) / \cosh (k h)$ avec $k$ le nombre d'onde, $h$ la hauteur 
d'eau totale et $z$ la hauteur du capteur par rapport au fond. Il existe une fréquence maximale ou fréquence de coupure $(f c)$ au-delà de laquelle $K p$ devient instable à cause des problèmes numériques associés à la résolution d'un système hyperbolique. En utilisant les recommandations de [14], la partie du spectre correspondant à une valeur supérieure à $0,22 \mathrm{~Hz}$ a été donc supprimée $(K p \sim 0,1)$. L'énergie contenue dans cette partie est donc négligée pour l'estimation de la hauteur significative des vagues. L'application de $K p$ et l'utilisation de la théorie linéaire de la houle introduisent une sous-estimation de la hauteur des vagues de l'ordre de $15 \%$ [14]. Une fois le traitement spectral réalisé, la nature des différents pics de vagues (houle ou mer de vent) ainsi détectés est déterminée à partir de critères d'âge et de direction des vagues [1]. Ainsi, un pic sera attribué à un pic de mer vent si :

- $\quad c_{\text {houle }} / U_{\text {vent }}<c$ avec $\mathrm{c}=1,05, c_{\text {houle }}$ la vitesse de phase de la houle et $U_{\text {vent }}$ la vitesse du vent, $10 \mathrm{~m}$ au-dessus du niveau de la mer (critère d'âge).

- La différence de direction entre vagues et vent local est inférieure à $30^{\circ}$ (critère de direction)

\subsection{Formulation théorique de la mobilité sédimentaire 3.2.1. Mise en mouvement}

Pour qu'un sédiment soit mis en mouvement, la contrainte au fond doit être supérieure à une contrainte critique $\left(\tau_{b}>\tau_{b, c r}\right)$. La plupart des calculs de contrainte critique de mise en mouvement sont basés sur les travaux de Shields [13], défini par: $\theta_{c}=\tau_{b, c r} / g\left(\rho_{s}-\rho\right) D_{50}$, avec $g$ l'accélération de pesanteur, $\rho_{s}$ et $\rho$ les masses volumiques du sédiment et de l'eau, et $D_{50}$ la taille de sédiment médiane. Ce paramètre de Shields critique de mise en mouvement du sédiment est fonction du paramètre de mobilité $D_{*}$ tel que $D_{*}=(g(s-1) / v) D_{50}$ avec $v$ la viscosité cinématique de l'eau, et $s$ la densité relative du sédiment. Afin d'estimer les tailles maximales de grains mis en mouvement par l'action conjointe des courants et des vagues, nous utilisons la valeur du paramètre de Shields $\theta_{1,2}=\theta_{1}+\theta_{2}$ (somme des paramètres de Shields lié au courant d'une part et aux vagues d'autre part), et, par méthode inverse, déduisons la taille maximale des grains mis en mouvement sous cette contrainte. Chaque type de contrainte au fond résulte d'une contrainte liée à la rugosité de grain et d'une contrainte liée à la rugosité de peau (prise en compte de la présence de rides de fond). Pour la mise en mouvement, seules les contraintes de courants et vagues liées à la rugosité de peau sont à considérer $\left(z_{0}=k_{s} / 30=3 D_{50} / 30\right.$ avec $z_{0}$ la longueur de rugosité et $k_{s}$ la hauteur de rugosité). En terme de domaine granulométrique étudié, à la position $\mathrm{S} 4$, les observations [11,15] indiquent la présence dominante de sables fins à moyen $\left(0,063 \mathrm{~mm}<D_{50}<0,5 \mathrm{~mm}\right)$. Seuls les sédiments non-cohésifs sont donc considérés $\left(D_{50}>0,063 \mathrm{~mm}\right)$. 
Contraintes de courant $\tau_{b, c}$ : la mesure effectuée par le S4 donne la vitesse du courant à la cote $z=0,40 \mathrm{~m}$ au-dessus du fond. Il s'agit d'estimer la contrainte de courant au fond $\left(\tau_{b, c}\right)$ à partir de cette mesure. Des mesures de profils verticaux effectués dans la même zone, pendant la même période, montrent que la vitesse suit un profil logarithmique sur la verticale (colonne d'eau bien mélangée). Un profil de vitesse logarithmique peut donc être utilisé et, en utilisant la relation entre la contrainte au fond et la vitesse de frottement au fond, on obtient une relation directe entre la contrainte au fond et la mesure effectuée : $\tau_{b, c}=\rho \kappa^{2}\left(u(z, t) / \ln \left(z / z_{0}\right)\right)^{2}$ avec $\kappa$ la constante de von Kármán $(0,41)$.

Contraintes de vagues $\tau_{b, w}$ : la contrainte au fond due aux vagues $\left(\tau_{b, w}\right)$ et moyennée sur un demi-cycle de vagues, s'exprime sous la forme (van Rijn, 1989) : $\tau_{b, w}=0.25 \rho f_{w}\left(u_{b}\right)^{2}$ avec $u_{b}$ est la valeur pic de la vitesse orbitale près du fond, suivant la théorie linéaire. $f_{w}$ est le coefficient de frottement des vagues qui est fonction de la longueur d'excursion des vagues.

Flux de sédiments charriés : afin d'étudier l'importance du rôle de la houle dans le transport de sédiment, les flux de sédiments charriés sont calculés en utilisant la relation de van Rijn [13], qui est la relation la plus utilisée et reconnue en domaine off-shore, domaine où est effectuée la mesure S4.

$$
q_{b}(t)=0.25 \rho_{s} \alpha D_{50} D_{*}^{-0.3}\left[\tau_{c w}^{\prime} / \rho\right]^{0.5}\left[\left(\tau_{c w}^{\prime}-\tau_{b, c r}\right) / \tau_{b, c r}\right]^{1.5}
$$

Avec $\alpha=1-\left(H_{s} / h\right)^{0.5}, H_{s}$ la hauteur significative des vagues, $\tau_{c w}^{\prime}$ la somme des contraintes de peau de vagues et de courant et $\tau_{b, c r}$ la contrainte critique de mise en mouvement du sédiment..

\section{Hydrodynamique : résultats}

\subsection{Marée}

Après traitement de la mesure du courantomètre $\mathrm{S} 4$, nous obtenons le signal de marée (Fig. 2b), ainsi que les composantes de marée. La marée issue du courantomètre S4 est similaire à celle observée à La Rochelle (Fig. 2b), avec une amplitude du signal de marée de $10 \%$ plus faible que celle observée à La Rochelle, s'expliquant physiquement par le fait que la propagation de la marée est conditionnée par la forme du fond, la profondeur et la forme de la côte. Par rapport aux résultats du modèle de marée, au point de mesure, les premières composantes montrent un bon accord entre modèle SHOM et mesures S4, avec une différence relative de $0,23 \%$ pour la composante $\mathrm{M} 2$ et de $3 \%$ pour la composante S2.

\subsection{Courants de marée}

Le courant de marée obtenu après traitement (Fig. 2c), à $40 \mathrm{~cm}$ au-dessus du fond, est de $0,38 \mathrm{~m} / \mathrm{s}$ (jusant) en vive-eau et de $0,08 \mathrm{~m} / \mathrm{s}$ en morte-eau (jusant), avec une 
asymétrie flot-jusant (courant de flot plus faible et durée plus longue, par rapport au jusant) qui est d'autant plus forte que le coefficient de marée est faible. Pour le cycle de marée le plus proche d'une vive-eau moyenne pendant la campagne (le $8 / 11 / 2001$, coef. de marée de 92), les courants de flot et jusant, intégrés sur la verticale, sont respectivement de $0,41 \mathrm{~m} / \mathrm{s}$ et $0,48 \mathrm{~m} / \mathrm{s}$, ce qui est en accord avec les valeurs de courant, intégré sur la verticale, fournies dans l'atlas de courants [12] au niveau du seuil interinsulaire $(\sim 0,45 \mathrm{~m} / \mathrm{s}$ pour un coef. de 95$)$.

\subsection{Courants résiduels}

En pratique, les courants résiduels (signal brut moins signal haute fréquence, moins signal de marée) incluent les courants induits par le vent, les courants stationnaires induits par les vagues et les courants géostrophiques (négligeables dans la zone d'étude). Les mesures réalisées montrent que les courants résiduels obtenus (Fig. 2c) sont plus faibles que les courants de marée, mais peuvent atteindre ponctuellement le même ordre de grandeur (Fig. 2c). Ces courants résiduels semblent principalement résulter des courants induits par le vent, plutôt que des courants stationnaires induits par les vagues [6].
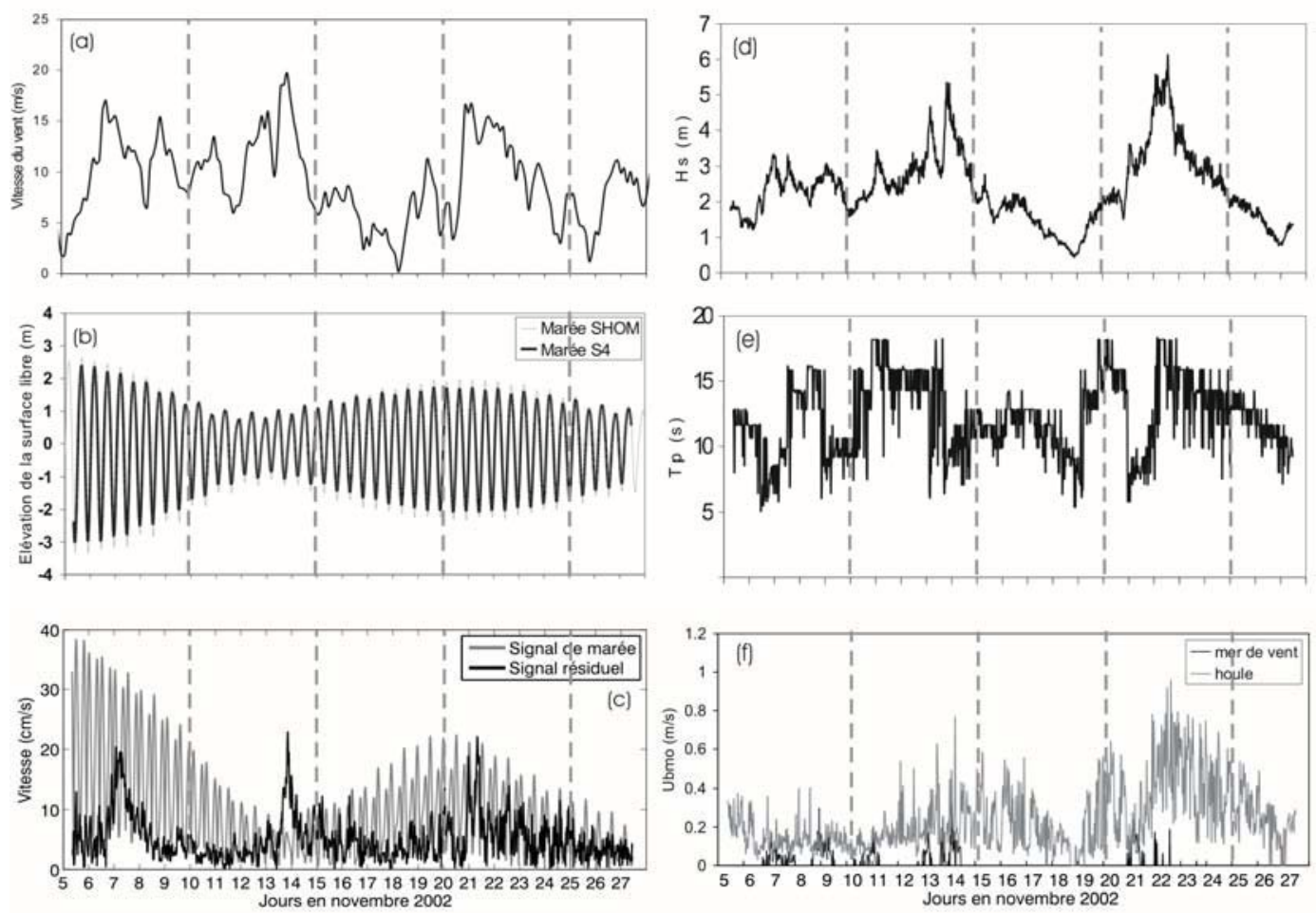

Figure 2 - Données et résultats hydrodynamiques. a : vitesse du vent au point $\left(46^{\circ} \mathrm{N} ; 2.5^{\circ} \mathrm{W}\right)$, modèle Wavewatch III. b : marée prédite (SHOM) à La Rochelle-Pallice et marée après analyse du $\mathrm{S} 4$. c : module du courant de marée ( $\mathrm{S} 4$ analysé) et courant résiduel près du fond. $\mathrm{d}$ : hauteur significative des vagues (S4). e : période pic des vagues (S4). $\mathrm{f}$ : vitesse orbitale des houles et mer de vent, théorie linéaire (S4). 


\subsection{Vagues}

Les mesures (pression-vitesse) montrent que, pendant la campagne, la période pic moyenne des vagues est de l'ordre de $12 \mathrm{~s}$, la direction pic moyenne est d'environ $260^{\circ}$ (en provenance d'Ouest) et la hauteur significative atteint plus de $6 \mathrm{~m}$ le 22/11 (Fig. 2d, 2e). Notons que les périodes pic et hauteurs significatifs sont obtenues par traitement de tout le spectre de vagues (houle et mer de vent). En outre, la figure $2 \mathrm{f}$ montre que les vitesses orbitales dues à la houle sont largement supérieures à celles liées à la mer de vent, et donc sont dominantes en terme de dynamique sédimentaire liée aux vagues.

\section{Mobilité sédimentaire : résultats}

\subsection{Mise en mouvement}

Les courants de marée seuls sont suffisants pour mettre en mouvement des sables (taille maximale de $0,5 \mathrm{~mm}$ ), uniquement en période de vive-eau (Tab. 1, $\mathrm{D} 50 \mathrm{Cm})$. Le courant résultant de la superposition des composantes de marée et résiduelles engendre une mise en mouvement de sédiments légèrement plus grossiers (jusqu'à $0,57 \mathrm{~mm}$ ) (Tab. 1, Fig. 3, D50C), pendant seulement $4 \%$ de la période de mesure. Par contre, les vagues seules mettent en mouvement les sédiments pendant quasiment toute la période de mesure (Tab. 1, D50V), avec une mise en mouvement des grains de $0,2 \mathrm{~mm}$ pendant $90 \%$ du temps. La taille moyenne des sédiments alors mis en mouvement est de $1,3 \mathrm{~mm}$, tandis que la taille maximale est de $5,3 \mathrm{~mm}$. L'action conjointe des courants (totaux) et des vagues modifie peu les caractéristiques de mise en mouvement pendant la période de mesure (Tab. 1, Fig. 3, D50CV) avec une durée de mise en mouvement des grains de $0,2 \mathrm{~mm}$ pendant $92 \%$ du temps, les périodes de non-mise en mouvement correspondant aux périodes d'accalmie (e.g. : 18 nov.). Ceci montre l'action prépondérante des vagues dans la mise en mouvement du sédiment.

\begin{tabular}{|l|l|l|l|l|}
\hline Courbes (Fig. 3) & D50Cm & D50C & D50V & D50CV \\
\hline Courants de marée & $\mathrm{x}$ & $\mathrm{x}$ & - & $\mathrm{x}$ \\
\hline Courants résiduels & - & $\mathrm{x}$ & - & $\mathrm{x}$ \\
\hline Vagues & - & & $\mathrm{x}$ & $\mathrm{x}$ \\
\hline Mobilité* des grains de 0,2 $\mathrm{mm}$ & $3 \%$ & $4 \%$ & $90 \%$ & $92 \%$ \\
\hline Taille de grain maximale & $0,5 \mathrm{~mm}$ & $0,57 \mathrm{~mm}$ & $5,3 \mathrm{~mm}$ & $5,3 \mathrm{~mm}$ \\
\hline Taille de grain moyenne & $0,07 \mathrm{~mm}$ & $0,08 \mathrm{~mm}$ & $1,3 \mathrm{~mm}$ & $1,3 \mathrm{~mm}$ \\
\hline
\end{tabular}

Tableau 1. Tailles de grain obtenues en fonction des processus hydrodynamiques. Mobilité : rapport entre durée de mise en mouvement et durée de la campagne.

\subsection{Transport}

Afin d'estimer le rôle respectif des vagues et des courants sur le transport de sédiments, les flux de sédiments sont estimés pour une taille de grain de $0,2 \mathrm{~mm}$ représentative de la zone (Fig. 3c). En ne prenant en compte que les courants (totaux), le flux moyen de sédiments sur la période de la campagne de mesure est de $0,0004 \mathrm{~kg} / \mathrm{m} / \mathrm{s}$, pour un flux maximal de $0,014 \mathrm{~kg} / \mathrm{m} / \mathrm{s}$. La prise en compte 
conjointe des vagues et des courants donne un flux solide moyen de $0,15 \mathrm{~kg} / \mathrm{m} / \mathrm{s}$ pour un flux maximal est de $2,40 \mathrm{~kg} / \mathrm{m} / \mathrm{s}$. Avec un flux de sédiment moyen pendant la campagne de mesure 370 fois plus grand, l'action des vagues apparait prépondérante dans la dynamique sédimentaire de la zone étudiée.

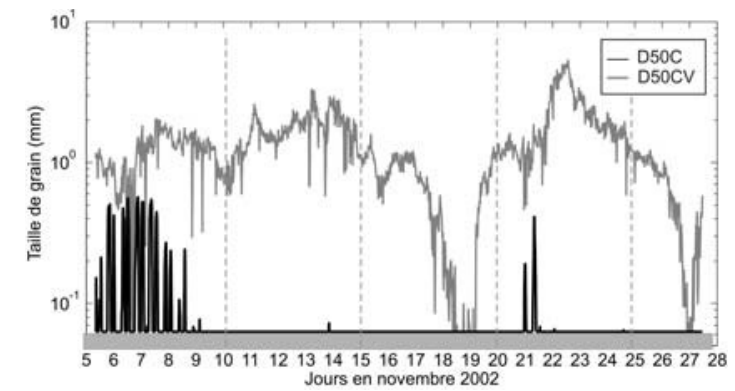

(a)

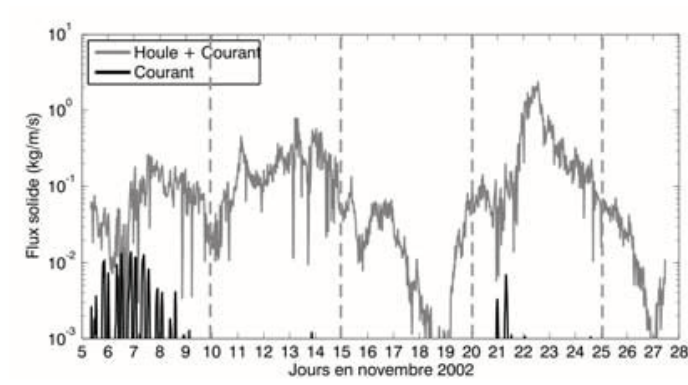

(b)

Figure 3 - Mobilité sédimentaire. a : Taille des grains mis en mouvement par les courants et les vagues (D50C - courants totaux; D50CV - courants totaux et vagues). La zone grisée correspond aux sédiments non-cohésifs (non traité dans cette étude). $\mathrm{b}$ : Flux de sédiment.

\section{$\underline{\text { 6. Conclusion }}$}

La présente étude met en évidence l'importance de la houle dans la dynamique d'une plateforme continentale interne comme le site du Pertuis d'Antioche. A partir de mesures de pression et courant et d'une chaîne de traitement mise en place pour dissocier les différents agents hydrodynamiques et leur influence sur la dynamique sédimentaire, on montre que, par $23 \mathrm{~m}$ de fond, la dynamique sédimentaire est principalement contrôlée par la houle. La houle met en mouvement les sédiments, et les courants de marée et induits par le vent les transportent. Ceci confirme les résultats et hypothèses de [2] et [15], en dissociant de manière quantitative les courants de marée, courants résiduels, vitesses orbitales, et en estimant les tailles de grain potentiellement transportées. Pendant la campagne de mesure, les sables de 0,2 $\mathrm{mm}$ auraient ainsi été transportés pendant $92 \%$ du temps. Les périodes de vive-eau, en conjonction avec des périodes de tempête et forte houle, sont suffisamment énergétiques pour permettre le transport de sables moyens, voire de graviers (de taille $5 \mathrm{~mm}$ ).

\section{Remerciements}

Nous remercions les équipes de développement de $T_{-}$Tide et Diwasp, le SHOM pour mise à disposition de données, ainsi que E. Chaumillon, A. de Resseguier, et L. Choppin qui ont contribué aux observations in-situ. La campagne de mesure a été réalisée grâce au navire L'ESTRAN de l'université de La Rochelle.

\section{Références}

1 Aarnes J.E. and Krogstad H.E., (2001), Partitioning sequences for the dissection of directional ocean wave spectra: A review. Part of work package 4 (Wp4) of the EnviWave (EVG-2001-00017) research programme under the EU Energy, Environment and Sustainable Development programme. 
2 Barthe X. et Castaing P., (1989), Etude théorique de l'action des courants de marée et des houles sur les sédiments du plateau continental du Golfe de Gascogne. Oceanologica Acta 12(4), 325-334.

3 Bertin X., Chaumillon E., Sottolichio A. and Pedreros R., (2005), Tidal inlet response to sediment infilling of the associated bay and possible implications of human activities: the Marennes-Oléron Bay and the Maumusson Inlet, France, Continental Shelf Research, 25, 1115-1131.

4 Gerling T.W., (1992), Partitioning sequences and arrays of directional wave spectra into component wave systems. J. Atmos. And Oceanic Technol., 9, 444-458.

5 Hashimoto N., Nagai T. and Asai T., (1993), Modification of the extended maximum entropy principle for estimating directional spectrum in incident and reflected wave field. Rept. Of P.H.R.I., 32(4), 25-47.

6 Idier D., Pedreros R., Oliveros C., Sottolichio A., Choppin L. et Bertin X., (2006), Contributions respectives des courants et de la houle dans la mobilité sédimentaire d'une plateforme interne estuarienne. Exemple: le seuil interinsulaire, au large du Pertuis d'Antioche, France. C.R. Geoscience, sous presse.

7 Johnson D., (2002) DIWASP, a directional wave spectra toolbox for MATLAB ${ }^{\circ}$ : User Manual. WP 1601 DJ (V1.1), i-18. Centre for Water Research, University of Western Australia.

8 Le Roy R. et Simon B., (2003), Réalisation et validation d'un modèle de marée en Manche et dans le Golfe de Gascogne, Application à la réalisation d'un nouveau programme de réduction des sondages bathymétriques, Rapport d'étude SHOM 002/03.

9 Niedoroda A.W., Swift D.J.P. and Hopkins T.S., (1985), The shoreface, in Davis, R.A. Jr., Coastal Sedimentary Environments, Springer-Verlag, p. 533623.

10 Pawlowicz R., Beardsley B. and Lentz S., (2002), Classical Tidal Harmonic Analysis Including Error Estimates in MATLAB using T_TIDE, Computers and Geosciences, 28, 929-937.

11 SHOM, (2000), Carte spéciale G n6333G.

12 SHOM, (2001), Atlas de courants de marée : Côte Ouest de France, de Saint Nazaire à Royan, réf. 559-UJA. ISSN 1278-3684.

13 Van Rijn L.C., (1989), Handbook of sediment transport by currents and waves. Report H461, WL|Delft Hydraulics, The Netherlands.

14 Van Rijn, L.C., Grasmeijer, B.T and Ruessink, B.G, (2000), COAST3D Measurement errors of instruments for velocity, wave height, sand concentrations and bed levels in field conditions. Dep. of Physical Geography; University of Utrecht, WL Delft Hydraulics reports, $47 \mathrm{p}$.

15 Weber N., Chaumillon E., Tesson M. and Garlan T., (2004), Architecture and morphology of the outer segment of a mixed tide and wave-dominated-incised valley, revealed by HR seismic reflection profiling: the paleo-Charente River, Marine Geology, 207(1-4), p. 17-38. 